А.Ж. Сарымсакова ${ }^{1}$, А.Т. Ниязалиева ${ }^{1}$

${ }^{1}$ КГУСТА им. Н. Исанова, Бишкек, Кыргызская Республика

\author{
A.J. Sarymsakova ${ }^{1}$, A.T. Nijasalieva ${ }^{1}$ \\ ${ }^{1}$ KSUCTA n.a. N. Isanov, Bishkek, Kyrgyz Republic \\ (aelita.65@mail.ru, a-nijasalieva@mail.ru)
}

\title{
DIE FÖRDERUNG DER REZEPTIVEN FERTIGKEITEN DER TEXTARBEIT IN DER PRAXIS DEUTSCHUNTERRICHT
}

\section{РАЗВИТИЕ РЕЦЕПТИВНЫХ НАВЫКОВ РАБОТЫ С ТЕКСТОМ НА УРОКЕ НЕМЕЦКОГО ЯЗЫКА}

Бул макалада немис тили сабагында тексттер менен иштоонун машыгуу ыкмалары берилди. Студенттердин коммуникациялык компетенттүҮлүгүн өнүктүрҮҮ максатында, иш жүзундө текстти натыйжалуу пайдалануу сунушталат. Текст - тилдик бирдикти актуалдаштыруучу сүйлөө формасы жана рецептивдик жондомунун негизи катары каралат.

Өзөк сөздөр: коммуникативдик компетенциия, чет тилдеги текст, маңъыздын интеграциясы, тандалма/деталдуу/глобалдуу түшүнүҮ.

В статье рассматриваются эффективное использование текста в практике преподавание немецкого языка с целью развития коммуникативной компетенции студентов. Текст рассматривается как форма речевой актуализации языковых единиц, $u$ как основа для формирования соотносимых умений в рецептивных навыков речевой деятельности.

Ключевые слова: коммуникативная компетенция, иноязычный текст, интеграция смысла, соотносимые умения, выборочная/детальная/глобальная понимания.

The article discusses the effective use of text in the practice of teaching the German language in order to develop the communicative competence of students. The text is considered as a form of speech actualization of linguistic units, and as a basis for the formation of correlated skills in the receptive skills of speech activity.

Key words: communicative competence, foreign language text, sense integration, correlating skills, selective / detailed / global understanding.

Die Szene ist hinreichend bekannt: Die Bücher werden geöffnet und der Lehrer fordert einen Studenten auf, den ersten Satz eines neuen Abschnitts oder Kapitels zu lesen. Nach der Klärung unbekannter Wörter und Konstruktionen wird der Satz übersetzt; grammatische Fragen werden geklärt und Übersetzungsalternativen besprochen.

Ist der Lehrer mit dem Ergebnis zufrieden, bearbeitet ein anderer Student den zweiten Satz nach dem gleichen Schema. Es folgt der nächste Satz...

Am Ende des Abschnitts - vielleicht nach 25 bis 30 Minuten - versucht der Lehrer, mit den Studenten ein Gespräch über das Gelesene zu führen, doch im Auditorium herrscht betrenetes Schweigen; nur der Klassenprimus hat etwas anzumerken. Die anderen Studenten haben nichts verstanden, was wiederum der Lehrer nicht versteht.

So verlief meist unser Unterricht.

Darum sollen wir im Folgenden gehen: die Gründe zu erkennen, warum wir bei der oben skizzierten Methode der Texterarbeitung nicht verstanden, und die Grundlagen einer effektiven Art des Textverstehens in der Fremdsprache darzustellen. 
Beim Verstehen wirken zwei Faktoren zusammen: Umweltreize und das verstehende Subjekt. Eine einfache Theorie des Verstehens könnte etwa so lauten: «Die Umweltreize determinieren das Verstehen».

Um einen Text zu verstehen, braucht man nicht alle Daten gleichermaßen aufzunehmen und vollständig zu dekodieren. In der Tat hat die Leseforschung gezeigt, dass sich die Augen beim Lesen nicht kontinuierlich, sondern in Sprüngen fortbewegen und nur am bestimmten "Fixationspunkten» Halt machen.

Beim sprachlichen Verstehen werden also zwei Arten von Redundanzen wirksam: die in der Sprache selbst vollkommenden Redundanzen sowie all das, was wir aufgrund unseres sprachlichen und sonstigen Wissens an einen Text herantragen können und was den Text im Prozess des Verstehens "redundanz macht". Wolfgang Klein nennt folgende Arten von Informationen und Wissen, die beim sprachlichen Verstehen eine Rolle spielen:

- Sprachwissen: als Voraussetzung, Äußerungen in einer bestimmten Sprache zu tun und zu verstehen;

- Äußerungsinformation: “jene Information, die dem Wortlaut der Äußerung selbst gemäß den Regeln der betreffenden Sprache zu entnehmen ist".

- Kontextinformation: W.Klein unterscheidet drei Arten von Kontextinformationen:

- Weltwissens: enzyklopädisches Wissen, auch Wissen über (kulturspezifische) soziale Verhaltensnormen;

- Situationswissen: Informationen, die durch (visualle,akustische...) Wahrnehmung aus der Kommunikationssituation gewonnen werden;

- Vorgängerinformation: Informationen aus vorhergehenden sprachlichen Äußerungen und Kontexten.

Folgerungen für Didaktik des Textverstehens

In erster Linie befindet sich der Fremdspracheanwender in der Situation, verstehen zu müssen, obwohl er nicht versteht. Fremde Sprache, sei sie gelesen, gehört oder gesehen, kommt auf ihn $\mathrm{zu}$, und er muss sich verstehend behaupten. Dieses Verstehen-zum- Uberleben ist dem Schwimmen im tiefen Wasser vergleichbar, wo es darauf ankommt, den Kopf über Wasser zu halten und Land zu gewinnen. (Edelhoff, 1985).

"Verstehen - zum - Überleben" bezeichnet die Situation, in der sich jeder Fremdsprachensprecher außerhalb des Auditoriums befindet: Er ist gezwungen, fremdsprachige Texte "ohne Hilfe zu verstehen und das Verstandene zum Ausgangspunkt bzw. zur Grundlage außersprachlichen Verhaltens oder eigener Textproduktion zu machen”. (Solmecke, 1992).

Damit ist exakt das Lernziel des Textverstehenstrainings im Fremdsprachenunterricht bezeichnet. Die Verstehensschwierigkeiten von "Fremdsprachenanwendern" beruhen wesentlich auf ungenügender Sprachbeherrschung, doch damit sind nicht alle Gründe genannt. Zwei Grunde scheinen dafür verantwortlich zu sein: Offenbar ist es schwierig, die in der Muttersprache (weitgehend unbewusst) auflaufenden Rezeptionsverfahren auf den fremdsprachliche Verstehensprozess zu übertragen. Darüber hinaus begünstigt der Fremdsprachenunterricht selbst eine aufsteigende datengesteuerte Annährung an Texte, weil immer noch viel zu selten wissensgesteuerte Vertsehensstrategien vermittelt, sondern Texte meist Wort für Wort und Satz erarbeitet werden.

Vor allem eigentlichen Lesen oder Hören eines Textes sollen die Lernenden möglichst viele Informationen aktivieren, die sie dann in Form von wissensgesteuerten Prozessen an den Text herantragen und in der Interaktion mit dem Text für das Verstehen verwerten können. Für aufsteigende datengesteuerte Verstehensprozesse sollten zunächst alle Textteile verwendet werden, die ohne Schwierigkeiten verstanden werden oder von denen zu erwarten ist, dass sie relativ leicht zu verstehen sind.

$\mathrm{Zu}$ letzteren gehören oft Zahlen, Eigennamen, geographische Begriffe, internationale Wörter, Produktbezeichnungen, Zeitangaben.

Die Lerner müssen also lernen, zwei Menge von Textelementen für das Verstehen zu nutzen: Hinweise, die es erlauben, ihr Vormissen zu aktivieren und es an den Text heranzutragen. 
Das Ziel der Leser/ Hörer-Text-Interaktion liegt vor allem darin, von Bekanntem und Verstandenem auf Unbekanntes bzw. Unverstandenes zu schließen, z.B:

- Hypothesen über den Textinhalt zu prüfen, z.B. aus der Situationskenntnis Hypothesen über den Inhalt eines Gesprächs;

- Textlücken zu schließen, z.B. nicht verstandene Textteile oder Wörter zu erschließen;

- Textinhalte zu antizipieren, z.B. aus dem bisherigen Textverlauf auf folgende Textteile zu schließen.

Das Textverstehen verläuft so in Form einer Interaktion von aktivierten Schemata und Inseln des Verstehens, von wissensgesteuerten abgesteigenden und datengesteuerten aufsteigenden Verstehensprozessen.

„Wir liefern um dem Text nicht aus, sondern nutzen ihn, soweit wir ihn brauchen“. ( Solmecke, 1992)

Das heißt wir nähern uns Texten mit einem bestimmten Verstehensziel, und davon hängt die Qualität des Verstehens ab. Das Ziel des Verstehens besteht darin, eine subjektive Verstehensabsicht zu befriedigen. Die Lernen den müssen also Verstehensziele formulieren der Fragen an einen Text stellen, die ihrem Interesse bzw. ihrem Informationsbedürfnis entsprechen. (Westhoff,1991)

In der Fremdsprachendidaktik werden oft die Ebene des Verstehens unterschieden: glovales, selektives und detaliesiertes Verstehen. Diese Begriffe, die nicht genau definiert sind, meinen etwa Folgendes:

Glovalverstehen: Worum es in einem Text inhaltlich geht, d.h. die zentralen Inhaltspunkte; auch die bekannten W-Fragen: Kommunikationspartner (Wer schreibt/ spricht mit wem?), Thema (worüber?), Kommunikationssituation (Wo/ Wann?), kommunikatives Ziel (Wozu?), Textsorte usw. Es handelt sich also um die pragmatischen Bedingungen des Textes und den Inhalt auf einer allgemeinen Ebene.

Selektives Verstehen: Damit ist eine mittlere Ebene des Textverstehens gemeint; einem Text werden entsprechend der Verstehenabsicht gezielt bestimmte Informationen entnommen; oft handelt es sich einfach um die wichtigsten Textinformationen. Im Unterricht wird bei authentischen Verstehenstexten oft ein selektives Verstehen in diesem Sinne angestrebt.

Detailverstehen: Wort-für-Wort-Verständnis; alle Aussagen des Textes werden bis auf die Ebene des Einzelwortes verstanden. Bis ins Detail werden vor allem Lerntexte erarbeitet, aber auch bestimmte Textsorten bei Verstehenstexten. Die Lernenden müssen erkennen, dass bei vielen Texten ein Wort-für-Wort-Verständnis nicht erforderlich ist. Das muss im Unterricht immer wieder thematisiert und begründet werden, denn Fremdsprachenlehrer sind oft nur daran gewöhnt, Texte Wort für Wort zu erarbeiten.

Textarbeit

Die bisherigen Ausführungen haben Konsequenzen für die Textarbeit, und zwar gleichmaßen für den Umgang mit Verstehenstexten und Lerntexten. Generell sollte sich die Textarbeit von der höheren Ebene des Textes zu den niedrigenen Ebenen hin bewegen. Die Unterscheidung zwischen Verstehen und Lernstehen ist vor allem im Anfangsunterricht sehr wichtig. Lerntexte werden aus didaktischen Gründen bis ins Detail erarbeitet, denn ihr Stoff soll für die aktive Sprachverwendung erarbeitet und eingeübt werden. Verstehentexte werden hingegen eingesetzt, um die Fertigkeit «Textverstehen» zu fördern. Hier hängt es, wie oben dargestellt, von der Textsorte und den subjektiven Verstehensabsichten ab, welche Verstehensziele jeweils angestrebt werden.

- Verstehen auf höheren Textebenen erleichtert das Verstehen auf niedrigeren Textebenen. So können die Lernenden beim wissengesteuerten Verstehen aufgrund des unmittelbaren Kontextes und ihrer anderen Text- bzw. Weltkenntnisse unbekannten Wortschatz und Verstehenslücken für das Textverständnis selbstständig erschließen. Das ist bei einer umgekehrten (aufsteigenden) Vorgesehensweise nur schwerlich möglich.

- Beginnt die Textarbeit auf den höheren des Textes, so kann auf einer beliebigen Ebene mit dem Verstehen. bzw. der Textarbeit aufgehört werden - z.B. beim selektiven Textverstehen. Nur so 
ist es überhaupt möglich, schon bei Anfängern anhand von authentischen Texten das Verstehen zu fördern.

Aufsteigende Textarbeit kann man im Rahmen eines Drei-Phasen-Modells der Textarbeit durchgeführt werden.

Drei-Phasen-Modell der Textarbeit bei Verstehenstexten

1.Aktivierungsphase/ Hinführungsphase (Vorbereitung des Textverstehens)

- Aktivierung des Vorwissens

- Formulierung von Verstehenszielen

- Aktivierung von Verstehensstrategien

2. Verstehensphase

Ein oder mehrere «Verstehensdurchgänge», die spiralformig zu einem zunehmend tiefen Textverständnis führen.

- Anwendung von Verstehensstrategien

- Einsatz von Verstehensaufgaben

3. Anschlussphase

- Kommunikative Anschlussaktivitäten; z.B. themengebundene Studentenäußerungen, eventuell vorbereitet durch Einführen und Üben von Redemitteln.

- selektive sprachbezogene Textauswertung, z.B. Wortschatz.

Zur Hinführungsphase gehören alle auf den neuen Text bezogenen Aktivitäten, die durchgeführt werden, bevor die Lernenden den Text das erste Mal hören oder lesen. Diese Unterrichtsphase ist aus verschiedenen Gründen sehr wichtig:

- Die Studenten werden zur Thema des neuen Textes hingeführt; dabei werden vor allem die pragmatisch-kommunikativen Faktoren des Textes geklärt: die außersprachliche Situation bei dialogischen Texten (Wer kommuniziert mit wem? Wo? Worüber?); das kommunikative Umfeld bei Texten der geschriebenen Sprache (Textumgebung, Textsorte, Gegenstand des Textes ..). Dadurch wird beim Lerner ein Vorwissen aufgebaut, das ein zielgerichtetes Verstehen des Textes ermöglicht.

- Während der Hinführungsphase kann das inhaltliche und sprachliche Vorwissen der Studenten in Bezug auf den neuen Text aktiviert werden. Dadurch wird nicht nur das Textverstehen vorbereitet, sondern es wird zugleich eine wichtige Voraussetzung für die Eingliederung des neuen Lehrstoffs in die bestehende sprachliche Wissenstruktur geschaffen.

- Falls erforderlich, wird der Text während der Hinführungsphase sprachlich vorentlastet; d.h. es wird der Wortschatz eingeführt, der für das Verständnis der wichtigsten Textinformationen beim ersten Hören oder Lesen unbedingt erforderlich ist.

- Die Hinführungsphase hat schließlich auch eine wichtige motivationspsychologische Funktion. Bei den Studenten soll eine Erwartungsshaltung in Bezug auf den neuen Text geschaffen werden, und deshalb sind in dieser Phase Aktivitäten günstig, durch die ein Vorwissen aufgebaut und Interesse an neuen Text geweckt wird.

\section{Literatur}

1. Günter Storch Deutsch als Fremdsprache - Eine Didaktik

Wilchelm Fink Verlag München 1999. S.117-212

2. Wolfgang Klein Zweitspracherwerb. Eine Einführung 2.,

Auflage Frankfurt/M: Athenäum.1987

3. Christoff Edelhoff Authentizität im Fremdsprachenunterricht

München: Hueber 1985

4. Gert Solmecke Ohne Hören kein Sprechen. Bedeutung und Entwicklung

des Hörverstehens im Deutschunterricht.

Fremdsprache Deutsch 7. 1992

5. Gerart Westhoff Didaktik des Leseverstehens. Strategien des

voraussagenden Lesens mit Übungsprogrammen

München: Hueber 1987 